\title{
Possible relation of air ion density anomalies with earthquakes and the associated precursory ionospheric perturbations: An analysis in terms of criticality
}

\author{
M. Hayakawa ${ }^{1,2,3}$, S. M. Potirakis ${ }^{4,1}$ and Y. Saito ${ }^{5}$ \\ ${ }^{1}$ Hayakawa Institute of Seismo Electromagnetics Co. Ltd. (Hi-SEM), UEC (University of Electro-Communications) \\ Alliance Center \#521, 1-1-1 Kojimacho, Chofu, Tokyo, 182-0026, Japan \\ ${ }^{2}$ UEC, Advanced Wireless \& Communications research Center, 1-5-1 Chofugaoka, Chofu, Tokyo, 182-8585, \\ Japan \\ ${ }^{3}$ Geoscent Technologies Inc., 2-8-11 Akasaka, Minato-ku, Tokyo, 107-0052, Japan \\ ${ }^{4}$ Department of Electrical and Electronics Engineering, University of West Attica, Campus 2, 250 Thivon and P. \\ Ralli, Aigalao, Athens, GR-12244, Greece \\ ${ }^{5}$ Japan Society of Ecology and Disaster Prevention (JSEDiP), 14-23 Higashi-Hashimoto 4-chome, Midori-ku, \\ Sagamihara, 252-0144, Japan \\ E-mail: hayakawa@hi-seismo-em.jp
}

\begin{abstract}
The air ion density observed at Sendai during a period of 10/02/2013 to 30/04/2016 (more than 3 years) has been analyzed in terms of natural time critical analysis. It is then found that the air ions exhibit criticalities at some specific time periods, as a signature of pre-earthquake (EQ) fracturing process in the lithosphere. So it is likely to conclude that this criticality analysis enables us to associate the criticality in ion density to an EQ as a general tendency. The detection of air ions at a particular place suggests that those ions may be a precursor to an EQ (magnitude greater than 5.0) occurring within a radius of $200 \mathrm{~km}$ from the ion observatory. However, based on a comparison with the corresponding VLF/LF sub-ionospheric propagation data, we cannot say anything about a certain relationship of those air ions with the lower ionospheric perturbation as seen by VLF/LF, because the temporal scale of ions is medium-termed, but VLF/LF perturbation is short-termed, which makes it extremely difficult to correlate between the two.
\end{abstract}

Key words: Air ion density; EQ precursor; Criticality; Natural Time analysis; ionosphere

\section{Introduction}

It seems to be a consensus that different electromagnetic phenomena do appear before an earthquake (EQ) as precursors [Molchanov and Hayakawa, 2008; Hayakawa, 2015; Sorokin et al., 2015; Ouzounov et al. (Eds), 2018]. Among many precursors reported so far, there are two phenomena which are already evident as EQ precursors: (1) Ionospheric perturbations (not only in the lower ionosphere with the use of 
subionospheric VLF/LF (very low frequency ( $\mathrm{f}=3-30 \mathrm{kHz}) /$ low frequency $(\mathrm{f}=30-300 \mathrm{kHz})$ ) propagation (Hayakawa et al., 2010; Hayakawa, 2011) but also in the upper F region with the use of ground-based ionosondes and TEC observations and also satellite observations (Liu, 2009; Liu et al., 2013; Pulinets and Boyarchuk, 2004; Sorokin et al., 2015), and (2) ULF/ELF (f=1-10 Hz) impulsive radiation (Molchanov et al., 2004; Molchanov and Hayakawa, 2008; Schekotov et al., 2013; Schekotov and Hayakawa, 2017). The correlations of these two phenomena with EQs have been statistically confirmed on the basis of long-term (at least several years) data analysis, while other promising candidates seem to need further statistical studies.

In this paper we focus on the first topic of ionospheric perturbations in the lower ionosphere and their generation mechanism. Though there have been published a lot of reports on the presence of seismoionospheric perturbations and discussions on how those ionospheric perturbations appear prior to an EQ as a pre-seismic effect (Pulinets and Boyarchuk, 2004; Molchanov and Hayakawa, 2008; Liperovsky et al., 2008; Sorokin et al., 2015; Ouzounov et al., 2018), the mechanism is poorly understood. So far there have been proposed a few hypotheses on seismo-ionospheric perturbations in the context of lithosphereatmosphere-ionosphere (LAI) coupling (Hayakawa et al., 2004; Molchanov and Hayakawa, 2008; Sorokin et al., 2015).

The first (but most recently proposed) is "Electrostatic" channel, in which stress-activated positive holes are generated on the Earth's surface and a large electric field is generated as its result (Freund, 2009). The second is "Chemical (and associated electric field)" channel (Pulinets, 2009), in which radon emanation is the main player. Radon produces cluster ions and then cloud formation occurs. The cluster ions/aerosol size particles cause a change in air conductivity and a humidity drop near the Earth's surface, finally leading to the ionospheric perturbation. The third one is "Atmospheric gravity waves (AGW)" hypothesis [Hayakawa et al., 2011; Yang et al., 2019], in which water, radon and gas eruptions before an EQ could originate mosaic (in space) and twinkle (in time) spots of any ground parameters such as atmospheric pressure, temperature and density variations leading to the generation of AGW turbulence, which will produce turbulent variation in the lower ionosphere.

All of these three possible channels are still at the level of hypothesis, because it is impossible for us to have an extremely coordinated observation at any observing station. For example, we think that the third hypothesis plays the most important role in the LAI coupling with a lot of indirect and also some direct evidence in support to this channel [Hayakawa et al., 2011; Yang et al., 2019]. However, as indicated by 
[Liperovsky et al., 2008], it seems impossible to stress only one model, rejecting others. Whatever the mechanism among the three, the most important for us to to collect the information on what is happening on the Earth's surface and near-surface atmosphere before an EQ. So the information on geochemical measurements is of primary importance in the study of LAI coupling. Radon emanation has long been regarded as important for EQ prediction, and its measurements had been carried out extensively until around 1995 [e.g., King, 1986; Wakita, 1996], but regular observations of radon (either atmospheric or soil) as a target of EQ prediction becomes very scarce in recently years since the finding of radon emanation for the 1995 Kobe EQ [Igarashi et al., 1995; Yasuoka et al., 2010].

On the other hand, we comment on other geochemical precursors, and a good summary report has been published by [Biagi and Ermini, 2013]. The emanation of different gases has been performed in Kamchatka during a long period of 1999-2006. However, the regular (continuous) measurements of charged ions near the Earth's surface are very lacking, and have not attracted a lot of attention of scientists.

The present paper reports on the data of ion measurement at a particular observing site in Sendai, and we have used a new analysis concept of criticality based on the use of natural time (NT) analysis. Once we find criticality in the ion data, we are ready to forecast an impending EQ, so that we have first compared these criticalities in ion data with the actual EQs. Also, our VLF/LF data indicative of the ionospheric perturbation are presented in order to discuss, to some extent, the mechanism of LAI coupling. The EQ preparation process was based on the classical so-called seismic cycle, in which shortterm effects are explained in terms of dilatancy-diffusion model [Scholz, 1990]. Those classical simple models were recently reconsidered with the fast developing theory of fractals and chaos [Bak et al., 1988]. The concept of self-organized complex systems was put forward for EQ occurrence in [Rundle et al., 2000]. However, this approach using the pattern recognition techniques and self-organizing criticality, disregarded the physical and chemical changes happening in the lithosphere. Even in the field of seismoelectromagnetics, the importance of using these critical analyses has been recognized [Hayakawa et al., 1999; Gotoh et al., 2004; Smirnova et al., 2004; Ida et al., 2005]. In this paper, we apply the critical analysis to the ion data as a geochemical parameter, and we try to discuss the possible mechanism of emanation of ions in the context of LAI coupling.

In order to investigate the possible existence of any possible relation between the variations of ions in the air with significant EQs, we proceed here to the analysis of more than three years' measurements of 
air ion density using the recently proposed NT method [Varotsos et al., 2002, 2011a; Varotsos, 2005]. Following the successful application of the NT analysis to cases of EM phenomena appearing prior to significant EQs [Potirakis et al., 2013, 2016; Hayakawa et al., 2015a, 2015b], in this work we focus on the NT analysis of air ion density variations, because they were recorded at a ground-based ion observatory of Sendai, in Japan. Several time periods for which the air ion density variations present critical behavior are revealed, which seem to precede the occurrence of significant EQs. Moreover, a comparison with precursory low ionosphere perturbations reveals interesting similarities. Based on these similarities, a suggestion on the possible relation between the pre-EQ air ion density anomalies and the precursory ionospheric perturbations is discussed later for the first time.

The remaining of the article is organized as follows: Section 2 provides EQ information and describes the analyzed ion air density data along with the compared VLF/LF subionospheric propagation data; a brief description of the key concepts and basic formulas of the NT analysis method is provided in Section 3; the NT analysis of the ion air density is presented in Section 4; finally, the main findings are summarized in Section 5.

\section{Air ion density, EQ, and VLF subionospheric propagation data}

A brief description of our ion sensor is made here. We used commercially available equipment manufactured by AlphaLab Inc. (USA) to measure air (atmospheric) ion density. The instrument is a true ion density meter based on a Gerdien tube. Its intake is located at a height of about $3 \mathrm{~m}$ above the ground, and it contains a fun which pulls air through the meter at a calibrated rate. The air is fed to the air sensor with a guide of length of $2.5 \mathrm{~m}$ in an observation room. When the size of ions is greater than $1 \mu \mathrm{m}$, the sensor sensitivity becomes less. Only the positive ions are treated in this paper.

In this article we have used data for the variations of ion density in the air available from the air ion observatory of Sendai, Japan (Geographic coordinates: $38.05^{\circ} \mathrm{N}, 140.7^{\circ} \mathrm{E}$ ), belonging to the NPO Japan Earthquake Precursor Comprehensive Observation Center (JEPCOC, http://www.jsedip.jp). The air ion density was measured with a constant sampling rate of 1 sample per $20 \sec \left(f_{s}=0.05 \mathrm{~Hz}\right)$. Here the average air ion density values $\left\langle d_{i o n}\right\rangle$ (in $\mathrm{pcs} \cdot \mathrm{cm}^{-3}$ ) measured during the time period from 10/02/2013 to 30/04/2016 (3 years and 2 months) was analyzed. 
The obtained results were evaluated in the light of seismicity of a wide area around Japan during the same time period as retrieved from the Advanced National Seismic System (ANSS) Composite EQ Catalog available through the Northern California EQ Data Center (http://www.ncedc.org/anss/). Figure 1 illustrates the location of the Sendai ion observatory indicated by a black square, as well as the epicenters of all the EQs with $M_{W} \geq 5.0$ which took place in the time period of interest. The EQ depth is indicated by color code on the right of Figure 1.

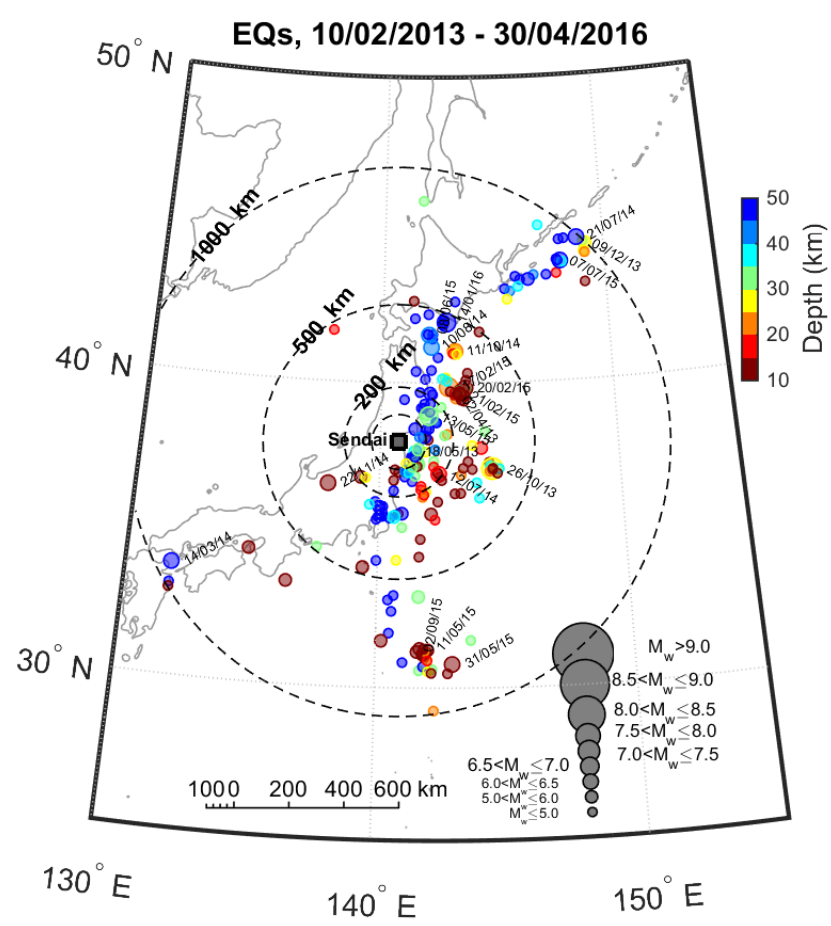

Figure 1: Map of the wider area around the Sendai ion observatory. The observatory position is indicated by the square, while all EQs with which happened with a depth shallower than $100 \mathrm{~km}$ are also shown for the time period from 10/02/2013 to 30/04/2016. The circle size is proportional to EQ's magnitude and its color refers to hypocenter's depth. The JST (UT+9) dates of the EQs with are also indicated, as well as indicative distances from the observatory.

The normalized "trend" $T R^{*}$ (average nighttime amplitude normalized with its standard deviation during preceding one month) of the subionospheric propagation for the path between the VLF transmitter JJY (located at Fukushima $\left.\left(37^{\circ} 22^{\prime} \mathrm{N}, 141^{\circ} 51^{\prime} \mathrm{E}\right), \mathrm{f}=40 \mathrm{kHz}\right)$ and the VLF/LF receiver of NSB (located at Nakashibetsu $\left(43^{\circ} 34^{\prime} \mathrm{N}, 144^{\circ} 58^{\prime} \mathrm{E}\right)$ during the time period from $01 / 11 / 2013$ to $30 / 04 / 2016$ were also employed. This propagation path has been chosen from our own VLF/LF network, because our ion 
station is located close to this path and is within the fifth Fresnel zone. According to the conventional nighttime fluctuation method [Hayakawa, 2011], a reduction of $T R^{*}$ to values below $-2 \sigma$ is a precursor of an impending EQ.

Finally, the index of local seismicity, $K l s$ which is a function of EQ magnitude, $M$, and epicentral distance (in $\mathrm{km}), R$, namely, $K l s=10^{0.75 \mathrm{M}} /(R+100)$ (Molchanov and Hayakawa 2008), was also employed. A higher $K l s$ value (at least greater than one) indicates higher "significance" of the EQ in relation to the observatory. Although this index has originally been proposed in order to interpret ultralow frequency (ULF) EM precursors, it provides, in any case, a means of quantifying the possible influence of an EQ preparation process to the measured EQ-influenced physical quantity by combining the factors of EQ magnitude with epicentral distance from the observatory. Since we expect both these factors to influence the "sensitivity" of an ion observatory to possibly EQ-related air ion density anomalies, we decided to use it in this study. Nevertheless, it is acknowledged that an adjustment of the above-mentioned $K l s$ formula is probably necessary for optimal use with air ion density observatory data; however this is beyond of the scope of the present article and will be addressed in the future.

Figure 2 portrays the employed air ion density data (Figure 2a), Kls for the EQs of Figure 1 (Figure $2 \mathrm{~b}$ ), as well as the $T R^{*}$ of the subionospheric propagation path JJY-NSB (Figure $2 \mathrm{c}$ ), in a common time axis over three years. In Figure 2a, the different segmentations of the air ion density time series for the NT analysis (see Section 4) are shown, while the dates for which criticality was found for the air ion density time series (see Section 4) are also depicted. Although not apparent from Figure 2a, the air ion density time series is dominated by some clear superposed (probably meteorological, geophysical (solar, lunar), seasonal) periodicities until the level of $\sim 100 \mathrm{pcs} \cdot \mathrm{cm}-3$. Up to this level, we consider air ion density variations as background. Moreover, some noticeable air ion density surges (spikes) stand out above this level reaching peak values as high as $\sim 3100 \mathrm{pcs} \cdot \mathrm{cm}-3$. In this study we focus on the NT analysis (see Section 4) on the air ion density values well exceeding the background, i.e., above 300 pcs $\cdot \mathrm{cm}-3$ (arbitrarily selected). 


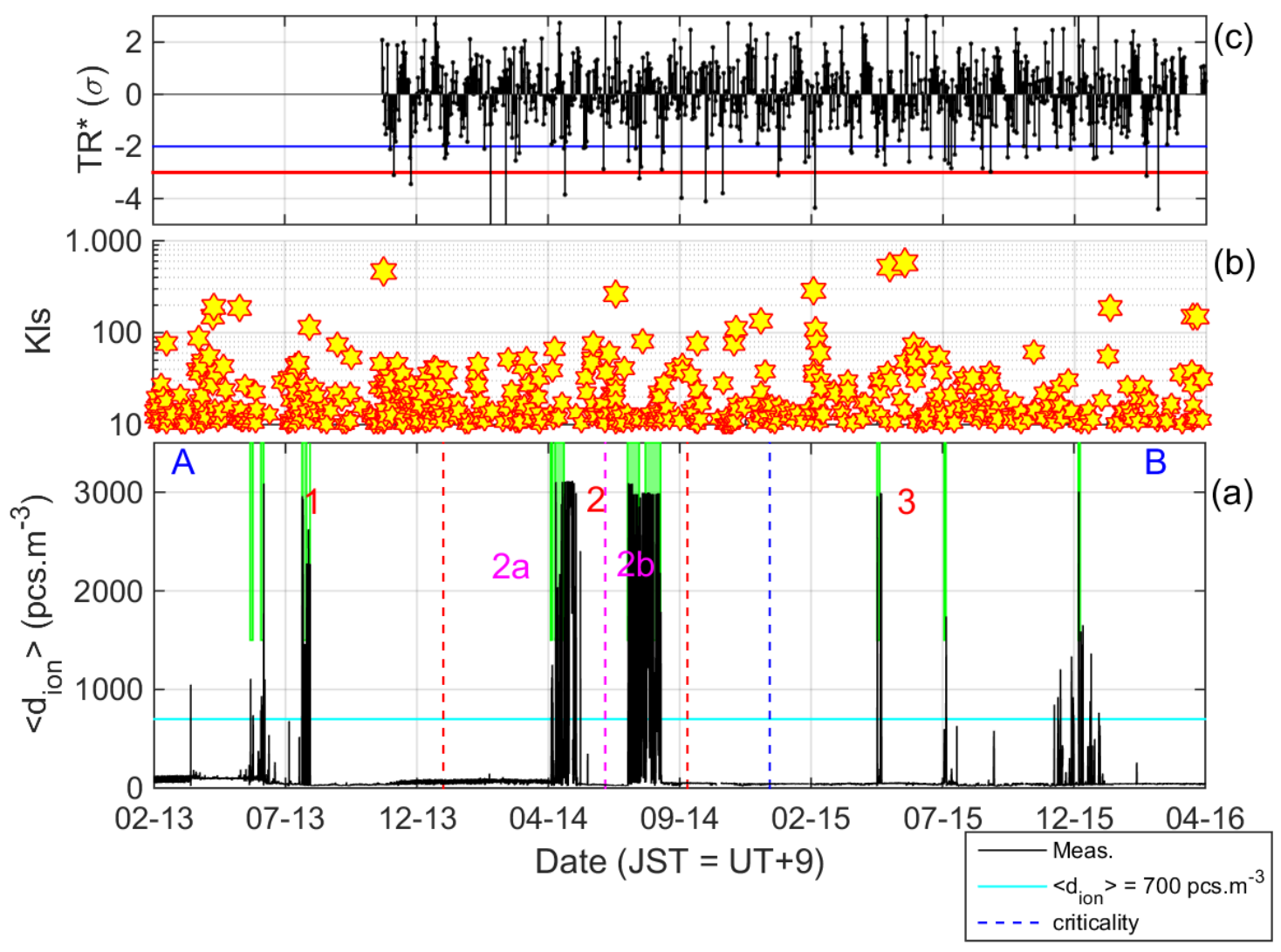

Figure 2: (a) Temporal variation of air ion density ; the horizontal cyan line defines an arbitrarily selected threshold of 700 pcs.cm-3 above which we consider the ion density "noticeably" high; the vertical dashed lines define the borders between the parts to which the whole time series was divided in order to form the different analyzed sub-series; the blue-colored divides the whole time series into parts "A" and "B", the red-colored into "1", "2" and " 3 ", while the magenta-colored subdivides " 2 " into " $2 a$ " and " $2 b$ " (for the analyzed cases see Table 1 and text in Section 4); the vertical "hanging" green-colored patches indicate that the NT analysis revealed criticality time periods. (b) Index of local seismicity, $\mathrm{z}$, for the EQs of Figure $1(\mathrm{M} \geq 5$, epicentral distance $\leq 1000 \mathrm{~km}$, Depth $<$ $100 \mathrm{~km}$ ). (c) Normalized trend, TR*, of the subionospheric propagation path JJY-NSB. Time axis is common in JST (UT+9).

\section{NT analysis method}

The NT method was originally proposed for the analysis of ULF $(\leq 1 \mathrm{~Hz})$ ) SES signals [Varotsos et al., 2002, 2005], and has been shown to be optimal for enhancing the signals in the time-frequency space [Abe et al., 2005]. The transformation of a time-series of "events" from the conventional time domain to NT domain is performed by ignoring the time-stamp of each event and retaining only their normalized order (index) of occurrence. Explicitly, in a time series of $N$ successive events, the natural time $\chi_{k}$ of the 
$K^{\text {th }}$ event is the index of occurrence of this event normalized by dividing by the total number of the considered events, $\chi_{k}=K / N$. On the other hand, the "energy", $Q_{k}$ of each $K^{\text {th }}$ event is preserved. We note that the quantity $Q_{k}$ represents different physical quantities for various time series: for EQ time series it has been assigned to a seismic energy released (e.g., seismic moment) [Varotsos et al., 2005], and for SES signals that are of dichotomous nature it corresponds to SES pulse duration [Varotsos, 2005], while for geophysical scale $\mathrm{MHz}$ EM signals that are of non-dichotomous nature, it has been attributed to the energy of fracto-EM emission events as defined in [Potirakis et al., 2013]. The transformed time series $\left(\chi_{k}\right.$ , $\left.Q_{k}\right)$ is then studied through the normalized power spectrum $\Pi(\varpi)=\left|\sum_{k=1}^{N} p_{k} \exp \left(j \varpi \chi_{k}\right)\right|^{2}$ where $\varpi$ is the natural angular frequency, $\varpi=2 \pi p$, with $\varphi$ standing for the frequency in NT, termed "natural frequency", and $\quad p_{k}=Q_{k} / \sum_{n=1}^{N} Q_{n}$ corresponds to the $K^{\text {th }}$ event's normalized energy. Note that the term "natural frequency" should not be confused with the rate at which a system oscillates when it is not driven by an external force; it defines an analysis domain dual to the NT domain, in the framework of Fourier-Stieltjes transform (Varotsos et al., 2011a).

The study of $\Pi(\varpi)$ at $\varpi$ close to zero reveals the dynamic evolution of the time series under analysis. This is because all the moments of the distribution of $p_{k}$ can be estimated from $\Pi(\varpi)$ at $\varpi \rightarrow 0$ (Varotsos et al., 2011b). Aiming to that, by the Taylor expansion $\Pi(\varpi)=1-\kappa_{1} \varpi^{2}+\kappa_{2} \varpi^{4}+\ldots$, the quantity $\kappa_{1}$ is defined, where $\kappa_{1}=\sum_{k=1}^{N} p_{k} \chi_{k}^{2}-\left(\sum_{k=1}^{N} p_{k} \chi_{k}\right)^{2}$, i.e., the variance of $\chi_{k}$ weighted for $p_{k}$ characterizing the dispersion of the most significant events within the "rescaled" interval . Moreover, the entropy in NT, $S_{n t}$, is defined [Varotsos et al., 2006] as $S_{n t}=\sum_{k=1}^{N} p_{k} \chi_{k} \ln \chi_{k}-\left(\sum_{k=1}^{N} p_{k} \chi_{k}\right) \ln \left(\sum_{k=1}^{N} p_{k} \chi_{k}\right)$ and corresponds [Varotsos et al., 2006, 2011a] to the value at $q=1$ of the derivative of the fluctuation function $f l(q)=\left\langle\chi^{q}\right\rangle-\langle\chi\rangle^{q}$ with respect to $q$ (while $\kappa_{1}$ corresponds to $f l(q)$ for $q=2$ ). It is a dynamic entropy depending on the sequential order of events [Varotsos et al., 2006]. The entropy, $S_{n t-}$, obtained upon considering [Varotsos et al., 2006] the time reversal $T$, i.e., $T p_{m}=p_{N-m+1}$, is also considered. 
A system is considered to approach criticality when the parameter $\kappa_{1}$ converges to the value $\kappa_{1}=0.070$ and at the same time both the entropy in NT and the entropy under time reversal satisfy the condition $S_{n t}, S_{n t-}<S_{u}=(\ln 2 / 2)-1 / 4$ [Sarlis et al., 2011], where $S_{u}$ stands for the entropy of a "uniform" distribution in NT [Varotsos et al., 2006]. It has to be mentioned that the criterion of the $\kappa_{1}=0.070$ value has originally been derived for SES activity and later on the basis of the Ising model. Its validity has been confirmed on real SES time series, while it has also been verified to be valid for several self-organized criticality models and real time series of a variety of applications. In all these dynamical systems, it has been found that the value $\kappa_{1}=0.070$ can be considered as quantifying the extent of the organization of the system at the onset of the critical stage [Varotsos et al., 2011a].

In the special case of NT analysis of foreshock seismicity [Varotsos et al., 2001, 2005, 2006; Sarlis et al., 2008], the seismicity is considered to be in a true critical state, a "true coincidence" is achieved, when three additional conditions are satisfied: (i) The "average" distance $\langle D\rangle$ between the curves of normalized power spectra $\Pi(\varpi)$ of the evolving seismicity and the theoretical estimation of $\Pi(\varpi)$, $\Pi_{\text {critical }}(\varpi)=\left(18 / 5 \varpi^{2}\right)-\left(6 \cos \varpi / 5 \varpi^{2}\right)-\left(12 \sin \varpi / 5 \varpi^{3}\right), \Pi_{\text {critical }}(\varpi) \approx 1-\kappa_{1} \varpi^{2}$, for $\kappa_{1}=0.070$ should be smaller than $10^{-2}$, i.e., $\langle D\rangle=\left\langle\left|\Pi(\varpi)-\Pi_{\text {critical }}(\varpi)\right|\right\rangle<10^{-2}$ (this is a practical criterion for signaling the achievement of spectral coincidence) (Varotsos et al., 2011a); (ii) the parameter $\kappa_{1}$ should approach the value $\kappa_{1}=0.070$ "by descending from above", i.e. before the main event the parameter $\kappa_{1}$ should gradually decrease until it reaches the critical value 0.070 (this rule was found empirically) [Varotsos et al., 2001, 2011a]; (iii) Since the underlying process is expected to be self-similar, the time of the true coincidence should not vary upon changing (within reasonable limits) either the magnitude threshold, $M_{\text {thres }}$, or the area used in the calculation.

It should be finally clarified that in the case of seismicity analysis, the temporal evolution of the parameters $\kappa_{1}, S_{n t}, S_{n t-}$, and $\langle D\rangle$ is studied as new events that exceed the magnitude threshold $M_{\text {thres }}$ are progressively included in the analysis. Specifically, as soon as one more event is included, first the time series $\left(\chi_{k}, Q_{k}\right)$ is rescaled in the NT domain, since each time the $k^{\text {th }}$ event corresponds to a NT 
$\chi_{k}=k / N$, where $N$ is the progressively increasing (by each new event inclusion) total number of the considered successive events; then all the parameters involved in the NT analysis are calculated for this new time series; this process continues until the time of occurrence of the main event.

Note that in the case of NT analysis of foreshock seismicity, the introduction of magnitude threshold, $M_{\text {thres }}$, excludes some of the weaker EQ events (with magnitude below this threshold) from the NT analysis. On one hand, this is necessary in order to exclude events for which the recorded magnitude is not considered reliable; depending on the installed seismographic network characteristics, a specific magnitude threshold is usually defined to assure data completeness. On the other hand, the use of various magnitude thresholds, $M_{\text {thres }}$, offers a means of more accurate determination of the time when criticality is reached. In some cases, it happens that more than one time-points may satisfy the rest of NT critical state conditions, however the time of the true coincidence is finally selected by the last condition that "true coincidence should not vary upon changing (within reasonable limits) either the magnitude threshold, $M_{\text {thres }}$, or the area used in the calculation."

\section{Analysis of the air ion density data}

Since this is the first attempt to analyze the ion density in the air using the NT method, the first step is to define the way of application of NT analysis and specifically the way that the notion of the "energy" $Q_{k}$ of the $k^{\text {th }}$ event, which is used in NT analysis (see Section 3), is defined. It has to be mentioned that air ion density is of non-dichotomous nature, while its sampling is quite fast (1 sample per $20 \mathrm{sec}$ ), as for example compared to the ULF characteristics corresponding to the magnetic field variation recorded prior to significant EQs (1 sample per day) which have also been analyzed using the NT method [Hayakawa et al., 2015a, 2015b; Potirakis et al., 2016]. Therefore, on one hand there is a need to use an appropriately selected threshold to define $Q_{k}$, while on the other hand it is practically impossible (mainly due to computer memory restrictions, but also in terms of processing time) to process the time series if each air ion density value above the threshold is considered an individual $Q_{k}$. Moreover, it was considered that it is reasonable to assume that when there were consecutive air ion density values exceeding the threshold these are possibly associated with each other (they are related with the same process). As a result, it was 
decided that the events $Q_{k}$ should be defined either as the sum of consecutive air ion density values exceeding the threshold, or unitary measurements (in case that there are no consecutive measurements exceeding the threshold at a specific time point). In other words, air ion density "energy" events $Q_{k}$ are defined in a way similar to the way that $Q_{k}$ has been defined for fracto-EM emission events in [Potirakis et al., 2013].

Summarizing the application of NT to the air ion density time series, it comprises the following steps: (a) We consider a sum of consecutive air ion density values exceeding a threshold, $d_{\text {ion,thres }}$, or a unitary air ion density value exceeding that threshold (in case that there are no consecutive measurements exceeding that threshold at a specific time point) as an event. The "energy" of $k^{\text {th }}$ event, that is the value of the quantity $Q_{k}$ of NT analysis (see Section 3), is considered to be equal to the corresponding sum of consecutive ion density values, or unitary ion density value (in case that there are no consecutive measurements exceeding that threshold at the specific time point). (ii) Then, the NT analysis is performed as in the case of pre-EQ seismic activity on the revealed "events". Starting from a specific time point, all the parameters $\left(\kappa_{1}, S_{n t}, S_{n t-},\langle D\rangle\right.$ defined in Section 3) are calculated for the time series of events rescaled in the NT domain each time a new event is added, checking for the corresponding criticality criteria as presented in Section 3 for the case of seismicity.

The next issue concerning the way of application of NT analysis to the air ion density time series has to do with the analyzed period. In the studied case, a quite long time period (> 3 years) is analyzed, while the possible relation of air ion density anomalies with EQs has not been adequately investigated in the literature [Bleier et al., 2013]. Therefore, it was decided that a "blind" analysis should be performed, i.e., the analysis should span the whole time period, not targeting a specific EQ or set of EQs. However, it is known that the NT analysis results depend on the start time of the analysis. Specifically, if consecutive criticality states are embedded in the same long time series, then preceding approaches to criticality might cause "masking" of possible following critical behavior. Since this is a blind analysis intending to cover the whole time period for which air ion density data are available, we decided to divide the total length of the time series to sub-series in an arbitrary way, which, however, would account for the noticeably high ion density spikes dominating at specific time periods. Therefore, it was decided that the following cases (indicated in Figure 2a, also see Table1) should be analyzed: 
(i) The total length time series

(ii) Division of the total length to two successive parts: (A) from the start (10/02/2013) to 31/12/2014 and

(B) from 01/01/2015 to the end (30/04/2016).

(iii) Division of the total length to three successive parts: (1) 10/02/2013 - 31/12/2013; (2) 01/01/2014 30/09/2014; (3) 01/10/2014 - 30/04/2016.

(iv) Further division of the above part (2) into two sub-parts: (2a) 01/01/2014 - 30/06/2014 and (2b) 01/07/2014 - 30/09/2014.

The obtained results of possible approach to criticality for each one of the above cases are summarized in Table 1, while their union also appears in Figure 2a (see green hanging background bars). It is noticeable from Table 1 that the criticality revealed for the time period 05-13/05/2014 in the total length case (case (i) above) "masks" any other criticality characteristics, indicating that was a correct strategy to analyze both total-length time series as well as different cases of sub-series. Comparing the similarity of the results as well as accounting for the possibility of "masking", we decided to accept the union of these periods as possible dates for criticality to be further used in our analysis.

Table 1: Summary of the possible dates for which the total-length air ion density time series or its sub-series were found to reach criticality by means of NT analysis (see also Figure 2a). The abbreviation "CR" in a specific cell of the table indicates that for the time series mentioned on the top row cell of the same column, NT criticality conditions were found to be satisfied for the time period mentioned on the left-most cell of the same row. In case that for different time series slightly different, overlapping, criticality periods were found, the exact criticality for each one of them appears next to the abbreviation "CR".

\begin{tabular}{|l|c|c|c|c|c|c|c|c|}
\hline \multicolumn{1}{|c|}{ Case $\rightarrow$} & Total length & $\mathrm{A}$ & $\mathrm{B}$ & 1 & 2 & $2 \mathrm{a}$ & $2 \mathrm{~b}$ & 3 \\
\hline $\begin{array}{l}\text { Criticality } \\
\text { period } \downarrow\end{array}$ & $10 / 02 / 2013-$ & $10 / 02 / 2013-$ & $01 / 01 / 2015-$ & $10 / 02 / 2013-$ & $01 / 01 / 2014-$ & $01 / 01 / 2014-$ & $01 / 07 / 2014-$ & $01 / 10 / 2014-$ \\
& $30 / 04 / 2016$ & $31 / 12 / 2014$ & $30 / 04 / 2016$ & $31 / 12 / 2013$ & $30 / 09 / 2014$ & $30 / 06 / 2014$ & $30 / 09 / 2014$ & $30 / 04 / 2016$ \\
\hline $29 / 05 / 2013-$ & $\mathrm{CR}$ & $\mathrm{CR}$ & & $\mathrm{CR}$ & & & & \\
$01 / 06 / 2013$ & & & & & & & & \\
\hline $10 / 06 / 2013-$ & $\mathrm{CR}$ & $\mathrm{CR}$ & & $\mathrm{CR}$ & & & & \\
$13 / 06 / 2013$ & & & & & & & \\
\hline $26-$ & $\mathrm{CR}$ & $\mathrm{CR}$ & & $\mathrm{CR}$ & & & & \\
$27 / 07 / 2013-$ & $(26-$ & $(26-$ & & $(27-$ & & & \\
$31 / 07 / 2013$ & $31 / 07 / 2013)$ & $31 / 07 / 2013)$ & & $31 / 07 / 2013)$ & & & \\
\hline $04 / 08 / 2013$ & $\mathrm{CR}$ & $\mathrm{CR}$ & & $\mathrm{CR}$ & & & & \\
\hline $30 / 04 / 2014-$ & $\mathrm{CR}$ & $\mathrm{CR}$ & & & CR & CR & & \\
$01-$ & $(30 / 04-$ & $(30 / 04-$ & & & $(30 / 04-$ & $(30 / 04-$ & & \\
$02 / 05 / 2014$ & $02 / 05 / 2014)$ & $02 / 05 / 2014)$ & & & & & & \\
\hline
\end{tabular}




\begin{tabular}{|c|c|c|c|c|c|c|c|}
\hline $\begin{array}{c}05 / 05 / 2014- \\
13- \\
15 / 05 / 2014\end{array}$ & $\begin{array}{c}\text { CR } \\
(05- \\
13 / 05 / 2014)\end{array}$ & $\begin{array}{c}\text { CR } \\
(05- \\
13 / 05 / 2014)\end{array}$ & & $\begin{array}{c}\text { CR } \\
(05- \\
15 / 05 / 2014)\end{array}$ & $\begin{array}{c}\text { CR } \\
(05- \\
15 / 05 / 2014)\end{array}$ & & \\
\hline $\begin{array}{c}25 / 07 / 2014 \\
-07 / 08 / 2014\end{array}$ & & & & & & $\mathrm{CR}$ & \\
\hline $\begin{array}{c}14- \\
18 / 08 / 2014- \\
31 / 08 / 2014\end{array}$ & & & & $\begin{array}{c}\mathrm{CR} \\
(18- \\
31 / 08 / 2014)\end{array}$ & & $\begin{array}{c}\text { CR } \\
(14- \\
31 / 08 / 2014)\end{array}$ & \\
\hline $\begin{array}{c}30 / 04 / 2015- \\
03 / 05 / 2015\end{array}$ & & & CR & & & & $\mathrm{CR}$ \\
\hline $\begin{array}{c}14 / 07 / 2015- \\
16 / 07 / 2015\end{array}$ & & & CR & & & & CR \\
\hline $\begin{array}{c}11 / 12 / 2015- \\
13 / 12 / 2015\end{array}$ & & & CR & & & & $\mathrm{CR}$ \\
\hline
\end{tabular}

In order to check for any possible relation between the revealed criticality in air ion density time series and EQs, we proceeded to a common three-dimensional (3D) representation of all possible criticality periods together with the time periods of "noticeably" high air ion density $(>700 \mathrm{pcs} \cdot \mathrm{cm}-3)$ in parallel to the seismicity evolution (EQ magnitude, $M_{w}$, and corresponding local seismicity index, $K l s$ ) with time and the distance, $L_{h-s}$, between the epicenter and the air ion observatory. Two cases of such 3D sets of figures are depicted in Figure 3a-d, and Figure 4a-d; for $M_{W} \geq 5.0$ (i.e., all EQs appearing in Figure 1, and Figure 2c) and $M_{W} \geq 6.0$, respectively, with (a)-(d) corresponding to progressive inclusion of more distant EQs for $L_{h-s}=100,200,500,1000(\mathrm{~km})$. Unfortunately, a time series correlation analysis was not possible between the important quantities time series $\left(\left\langle d_{i o n}\right\rangle, M_{W}, K l s\right.$, criticality time periods, time periods of "noticeably" high air ion density) because of the very different properties of each time series. Specifically, $\left\langle d_{i o n}\right\rangle$ is a continuously valued, uniformly sampled, time series (with relatively high sampling frequency), $M_{W}, K l s$, are also continuously valued time series, however with much lower number of samples appearing at random time points, while criticality time periods, and time periods of "noticeably" high air ion density $(>700 \mathrm{pcs} \cdot \mathrm{cm}-3)$ are binary valued time series. 


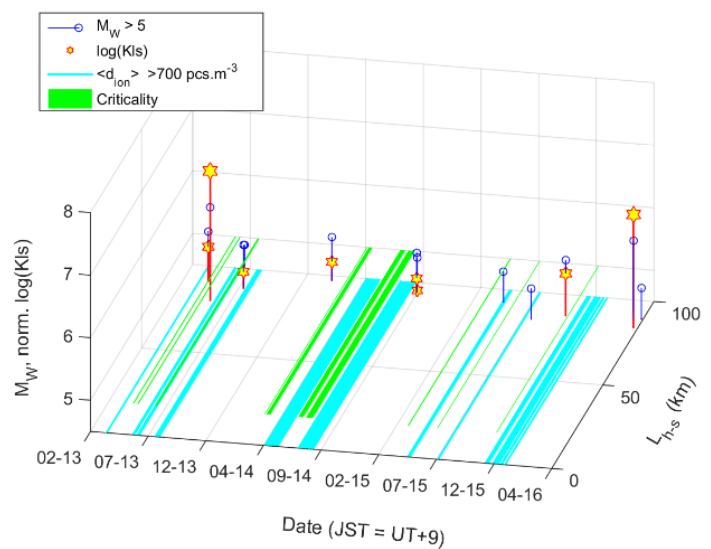

(a)

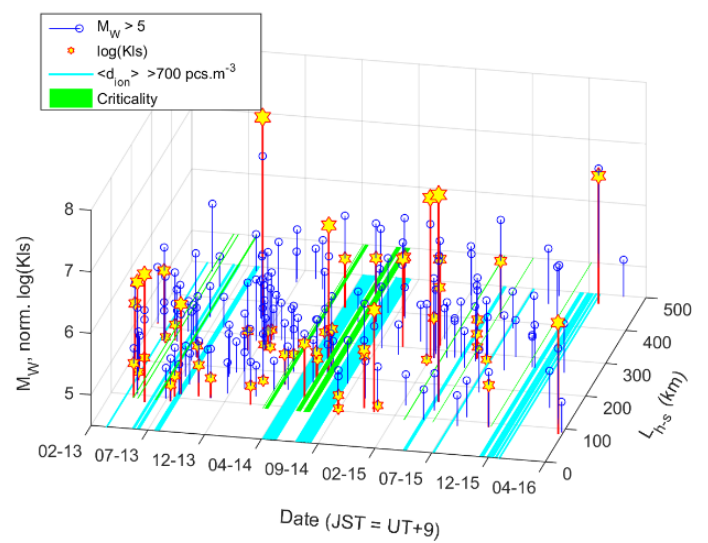

(c)

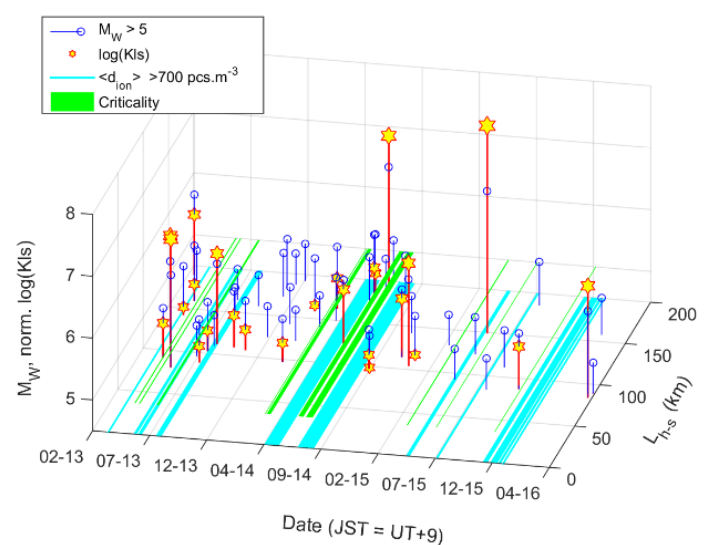

(b)

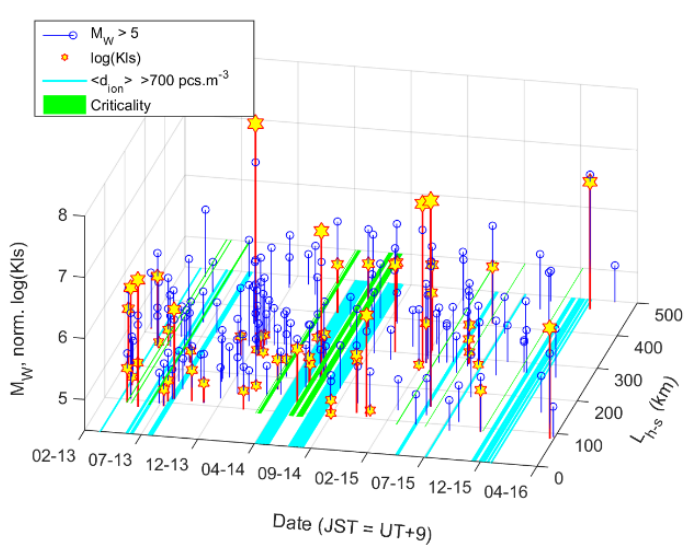

(d)

Figure 3: Temporal evolutions of the magnitude, $\mathrm{M}_{\mathrm{w}}$, and the corresponding logarithm of the local seismicity index (normalized) for the EQs appearing in Figure 1 with and progressive inclusion of more distant EQs; $\mathrm{M}_{\mathrm{w}} \geq 5.0$ : (a)(d) Criticality time periods revealed by NT method appear as horizontal green-colored patches, while the time periods of "noticeably" high air ion density $(>700 \mathrm{pcs} \cdot \mathrm{cm}-3)$ are also depicted as horizontal cyan-colored patches. Values of the normalized below 4.5 (corresponding to less significant EQs) do not appear.

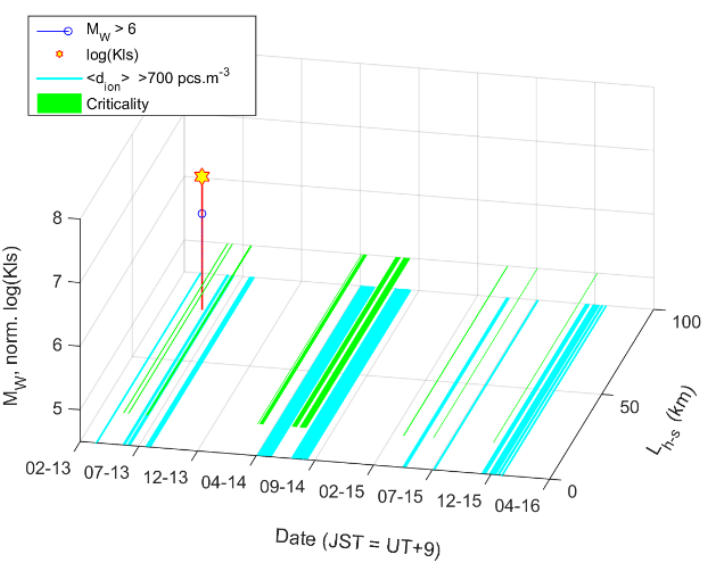

(a)

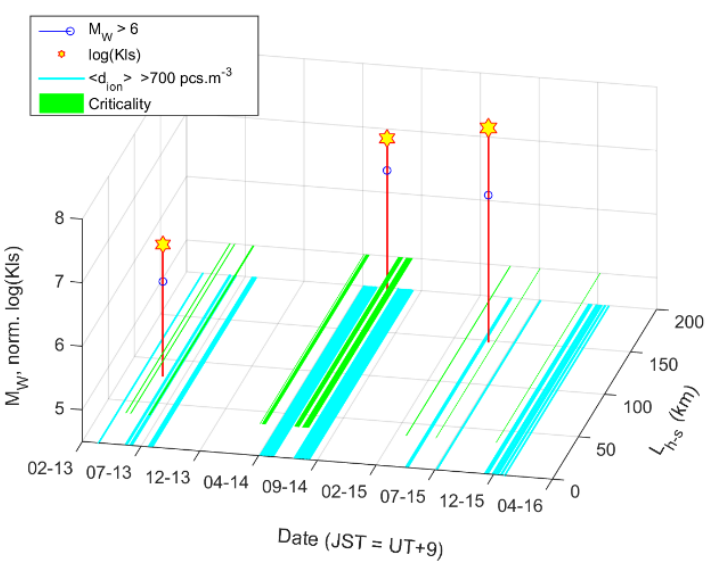

(b) 


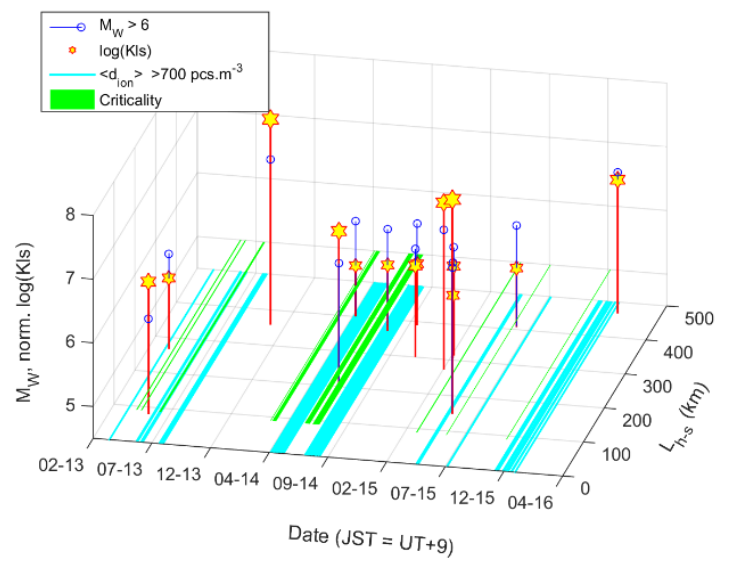

(c)

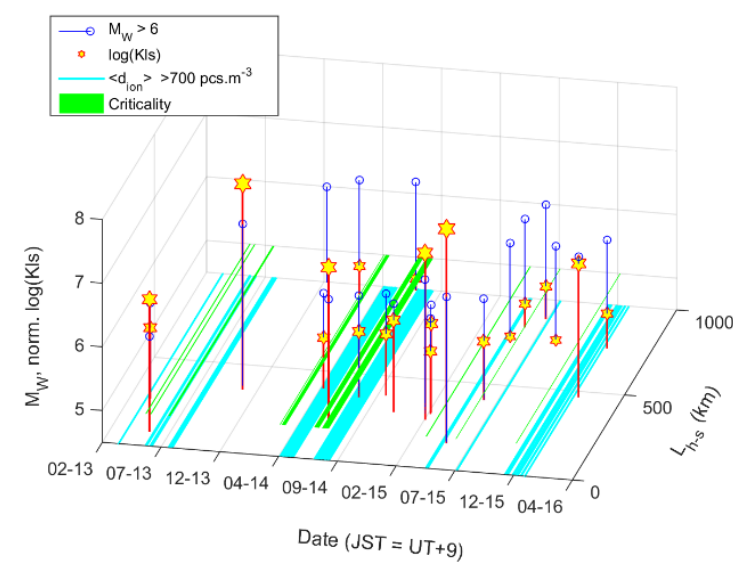

(d)

Figure 4: Temporal evolutions of the magnitude, $\mathrm{M}_{\mathrm{w}}$, and $\log (\mathrm{Kls})$ for the EQs appearing in Figure 1 with , as well as criticality time periods revealed by NT method and time periods of "noticeably" high air ion density $(>700$ pcs.cm-3).

In Figures 3 and 4 we have changed the covering distance of EQs with respect to the ion observatory: $\mathrm{L}_{\mathrm{h}-\mathrm{s}}=100,200,500$, and $1000 \mathrm{~km}$. When we look at Figures 3(c) and 3(d) and Figures 4(c) and 4(d), it seems that it is rather difficult to find any correlation between the criticality in ion densities and EQs. However, the situation changes significantly when the distance of Lh-s becomes smaller such as $200 \mathrm{~km}$ and $100 \mathrm{~km}$ as in Figures 3(a) and 3(b) for $M_{w} \geq 5.0$ and Figures 4(a) and 4(b) for $M_{w} \geq 6.0$. When $L_{h-s}=$ $100 \mathrm{~km}$ in Figures 3(a) and Figures 4(a), we cannot find any significant correlation of the criticality in ion densities with EQs. Even though we can observe three or four time intervals of criticality in ion density (green belts), no corresponding EQs seem to be detected. But when we increase $\mathrm{L}_{\mathrm{h}-\mathrm{s}}$ up to $200 \mathrm{~km}$ in Figures 3(b) and 4(b), there seems to exist a clear correlation between the two. In Figures 3(b) a group of EQs is likely to be associated with each of the criticality periods, and Figures 4(b) becomes more obvious between a big EQ with each criticality period. EQs are seen to take place just around the appearance time period of criticality in ion density. Hence, it may be concluded that the detection of air ions at a particular station is associated with EQs within a radius of $200 \mathrm{~km}$ from the ion observatory. In other words, the EQ preparation zone for air ions is, at least, $200 \mathrm{~km}$, so air ions can be ejected within this radius, or air ions are ejected near the EQ epicenter and followed by advection.

After having found some general association between the criticality in ion densities and EQs, we come back to Figure 2 and we try to look at the correlation between the criticality in ion density (green lines) and ion density itself to study the LAI coupling. It is known that the lead time of geochemical precursors 
is 20-50 days (Molchanov and Hayakawa, 2018), while that for the lower ionosphere (VLF/LF anomaly) is only about a week. We try to find any correlation between the VLF/LF anomalies and criticality conditions in ion density, but it seems to be unsuccessful because we are concerned with the general trends of both phenomena and also the temporal scales for both phenomena are different: medium-termed for air ions, but short-termed for VLF/LF anomaly. Hence, the ion detection as studied in this paper seems to be generally associated with EQs, but we cannot say any definite conclusion that air ions are the agent of LAI coupling, even though our initial intention of this paper was to obtain some positive answer to this question.

\section{Conclusion:}

The air ion density (positive) have been monitored in Sendai during a period of 10/02/2013 to 30/04/2016 (more than three years), and those data have been analyzed in terms of the critical NT method. The following findings have emerged from the present analysis.

(1) The NT analysis on ion data has indicated that the density of air ions have exhibited criticality, reflecting the pre-EQ fracturing process in the lithosphere.

(2) It is found from the general comparison of criticality periods of air ions with EQs that air ions are likely to be associated with an EQ with epicentral distance less than $200 \mathrm{~km}$. That is, the criticality in the lithosphere seems to be the origin of atmospheric air ions ejected within the EQ preparation zone.

(3) When we assume that air ions appear before an EQ, their precursory lead time seems to be on the order of one-two months, being consistent with previous result.

(4) A comparison of the criticality in ion density with the VLF propagation data suggests that we cannot say anything definite about the relation between the two, in contrast with our initial expectation as a possible candidate of air ions as the agent of seismo-lower ionospheric perturbation.

\section{Acknowledgments}

Two of the authors (S.M.P. \& M.H.) would like to express their gratitude to the Matsumae International Foundation for partly supporting this research by providing S.M.P. a 4-month research fellowship [Gno: 17G06] for visiting Hayakawa Institute of Seismo Electromagnetics Co. Ltd. Also thanks are due to the Northern California Earthquake Data Center and the Advanced National Seismic System (ANSS) for the 
access to the ANSS Composite Earthquake Catalog from which the EQs appearing in Figure 1 were retrieved.

\section{Reference}

Abe, S., Sarlis, N.V., Skordas, E.S., Tanaka, H.K., and Varotsos, P.A.,. Origin of the usefulness of the natural-time representation of complex time series, Phys. Rev. Lett., 94, 170601. doi: 10.1103/PhysRevLett.94.170601, 2005.

Bak, P., Tang, C., and Wiesenfeld, K., Self-organized criticality, Phys. Rev. A38, 364-374, 1988.

Biagi, P. F., and Ermini, A., Geochemical and VLF-LF radio precursors of strong earthquakes: A review, in "Earthquake Prediction Studies: Seismo Electromagnetics", Ed. by Hayakawa, TERRAPUB, Tokyo, 153-168, 2013.

Bleir, T., Dunson, C., Roth, S., Heraud, J., Lira, A., Freund, F., Dahlgren, R., Bambery, R., Bryant, N., Liu, J. Y., and Papadopoulos, G., Ground-based and space-based electromagnetic monitoring for preearthquake signals, in "Earthquake Prediction Studies: Seismo Electromagnetics", Ed. by Hayakawa, M., TERRAPUB, Tokyo, 113-127, 2013.

Gotoh, K., Hayakawa, M., Smirnova, N., and Hattori, K., Fractal analysis of seismogenic ULF emissions, Phys. Chem. Earth, 29, 419-424, 2004.

Fruend, F. T., Stress-activated positive charge carriers in rocks and the generation of pre-earthquake signals, in "Electromagnetic Phenomena Associated with Earthquakes", Ed. by Hayakawa, M., Transworld Research Network, Trivandrum, 41-96, 2009.

Hayakawa, M., Probing the lower ionospheric perturbations associated with earthquakes, Earthq. Sci., 24, 609-637, 2011.

Hayakawa, M., Earthquake Prediction with Radio Techniques. John Wiley \& Sons, Singapore, 294p, 2015.

Hayakawa, M., Itoh, T., and Smirnova, N., Fractal analysis of ULF geomagnetic data associated with the Guam earthquake on August 8, 1993, Geophys. Res. Lett., 26, 2797-2800, 1999.

Hayakawa, M., Kasahara, Y., Nakamura, T., Muto, F., Horie, T., Maekawa, S., Hobara, Y., Rozhnoi, A., Solovieva, M., and Molchanov, O. A., A statistical study on the correlation between the lower ionospheric perturbations as seen by subionospheric VLF/LF propagation and earthquakes, J. Geophys. Res., 115, A09305, doi:10.1029/2009JA015143, 2010. 
Hayakawa, M., Schekotov, A., Potirakis, S.M., Eftaxias K., Li, Q., and Asano, T., An integrated study of ULF magnetic field variations in association with the 2008 Sichuan earthquake, on the basis of statistical and critical analyses, Open J. Earthq. Res., 4, 85-93, doi: 10.4236/ojer.2015.43008, 2015a.

Hayakawa, M., Schekotov, A., Potirakis, S., and Eftaxias K., Criticality features in ULF magnetic fields prior to the 2011 Tohoku earthquake, Proc. Japan Acad. Ser. B, 91, 25-30. doi: 10.2183/pjab.91.25, $2015 b$.

Hayakawa, M., Molchanov, O. A., and NASDA/UEC team, Achievements of NASDA's Earthquake Remote Sensing Frontier Project, Terr. Atmos. Ocean. Sci., 15, 311-328, 2004.

Hayakawa, M., Kasahara, Y., Nakamura, T., Hobara, Y., Rozhnoi, A., Solovieva, M., Mochanov, O. A., and Korepanov, V., Atmospheric gravity waves as a possible candidate for seismo-ionospheric perturbations, J. Amos. Electr., 31, 129-140, 2011.

Ida, Y., Hayakawa, M., Adalev, A., and Gotoh, K., Multifractal analysis for the ULF geomagnetic data during the 1993 Guam earthquake, Nonlinear Process Geophys., 12, 157-162, 2005.

Igarashi, G., Saeki, S., Takahata, N., Sumikawa, K., Tasaka, S., Sasaki, Y., Takahashi, M., and Sano, Y., Ground-water radon anomaly before the Kobe earthquake in Japan, Science, 269, 60-61, 1995.

King, C. Y., Gas chemistry applied to earthquake prediction: an overview, J. Geophys. Res., 91(B), 12269-12281,1986.

Liperovsky, V. A., Pokhotelov, O. A., Mesister, C. V., and Liperoskaya, E. V., Physical models of coupling in the lithosphere-atmosphere-ionosphere system before earthquakes, Geomagnetsism and Aeronomy, 48(6), 795-806, 2008.

Liu, J. Y., Earthquake precursors observed in the ionospheric F-region, in "Electromagnetic Phenomena Associated with Earthquakes", Ed. by Hayakawa, M., Transworld Research Network, Trivandrum, p.187204, 2009.

Liu, J. Y., Chen, C. H., and Tsai, H. F., A statistical study on seismo-ionospheric precursors of the total electron content associated with $146 \mathrm{M} \geq 6.0$ earthquakes in Japan during 1998-2011, in "Earthquake Prediction Studies: Seismo Electromagnetics", Ed. by Hayakawa, M., TERRAPUB, Tokyo, p.17-30, 2013.

Molchanov, O.A., and Hayakawa, M., Seismo Electromagnetics and Related Phenomena: History and latest results, TERRAPUB, Tokyo, ISBN No.:978-4-88704-143-1, 189p, 2008.

Molchanov, O. A., Schekotov, A. Yu., Fedorov, E. N., Belyaev, G. G., Solovieva, M. S., and Hayakawa, M., Preseismic ULF effect and possible interpretation, Ann. Geophys., 47, 119-131, 2004. 
Ouzounov, D., Pulinets, S., Hattori, K., and Taylor, P., Pre-Earthquake Processes: A Multidisciplinary Approach to Earthquake Prediction Studies, Geophysical Monograph Series 234, AGU, Wiley, 365p. 2018.

Potirakis, S.M., Karadimitrakis, A., and Eftaxias, K., Natural time analysis of critical phenomena: the case of pre-fracture electromagnetic emissions, Chaos, 23, 023117. doi:10.1063/1.4807908, 2013.

Potirakis, S.M., Eftaxias, K., Schekotov, A., Yamaguchi, H., and Hayakawa, M., Criticality features in ultra-low frequency magnetic fields prior to the 2013 M6.3 Kobe earthquake, Ann. Geophysics, 59(3), S0317 (1-15), doi:10.4401/ag-6863, 2016.

Pulinets, S., Lithosphere-atmosphere-ionosphere coupling (LAIC) model, in "Electromagnetic Phenomena Associated with Earthquakes", Ed. by Hayakawa, M., Transworld Research Network, Trivandrum, p.235-253, 2009.

Pulinets, S. A., and Boyarchuk, K., Ionospheric Precursors of Earthquakes, Springer, Berlin, 315p, 2004. Rundle, J. B., Klein, W., Turcotte, D. L., and Malamud, B. D., Precursory seismic activation and criticalpoint change, Pure Appl. Geophys., 157, 2165-2182, 2000.

Sarlis, N.V., Skordas, E.S., Lazaridou, M.S., and Varotsos, P.A., Investigation of seismicity after the initiation of a Seismic Electric Signal activity until the main shock, Proc. Japan Acad. Ser. B, 84, 331343, 2008.

Sarlis, N.V., Skordas, E.S., and Varotsos, P.A., Similarity of fluctuations in systems exhibiting selforganized criticality, Europhys. Lett., 96(2). doi:10.1209/ 0295-5075/96/28006, 2011.

Schekotov, A., Fedorov, E., Molchanov, O. A., and Hayakawa, M., Low frequency electromagnetic precursors as a prospect for earthquake prediction, in "Earthquake Prediction Studies: Seismo Electromagnetics”, Ed. by Hayakawa, M., TERRAPUB, Tokyo, p.81-99, 2013.

Schokotov, A., and Hayakawa, M., ULF/ELF Electromagnetic Phenomena for Short-term Earthquake Prediction, LAP LAMBERT Acad. Publ., Beau Bassin, 102p, 2017.

Scholz, C. H., The Mechanics of Earthquakes and Faulting, Cambridge Univ. Press, Cambridge, 439p, 1990.

Smirnova, N., Hayakawa, M., and Gotoh, K., Precursory behavior of fractal characteristics of the ULF electromagnetic fields in seismic active zones before strong earthquakes, Phys. Chem. Earth, 29, 445-494, 2004.

Sorokin, V., Chmyrev, V., and Hayakawa, M., Electrodynamic Coupling of Lithosphere-AtmosphereIonosphere of the Earth, Nova Science Pub. Inc., New York, 326p, 2015.

Varotsos, P.A., The Physics of Seismic Electric Signals, TERRAPUB, Tokyo, 338p. 2005. 
Varotsos, P.A., Sarlis, N.V., and Skordas, E.S., Spatio-temporal complexity aspects on the interrelation between seismic electric signals and seismicity, Practica of Athens Academy, 76, 294-321, 2001.

Varotsos, P.A., Sarlis, N.V., and Skordas, E.S., Long-range correlations in the electric signals that precede rupture, Phys. Rev. E, 66, 011902. doi:10.1103/ PhysRevE.66.011902, 2002.

Varotsos, P.A., Sarlis, N.V., Tanaka, H.K., and Skordas, E.S., Similarity of fluctuations in correlated systems: The case of seismicity, Phys. Rev. E, 72, 041103. doi: 10.1103/PhysRevE.72.041103, 2005.

Varotsos, P.A., Sarlis, N.V., Skordas, E.S., Tanaka, H.K., and Lazaridou, M.S., Entropy of seismic electric signals: Analysis in the natural time under time reversal. Phys. Rev. E 73, 031114. doi:10.1103/PhysRevE.73.031114, 2006.

Varotsos, P.A., Sarlis, N.V., and Skordas, E.S., Natural Time Analysis: The New View of Time, Springer, Berlin, 245p, 2011a.

Varotsos, PA, Sarlis, N.V., Skordas, E.S., Uyeda, S., and Kamogawa, M., Natural time analysis of critical phenomena, Proc. Nat. Acad. Sci., USA, 108, 11361-11364. doi: 10.1073/pnas.1108138108, 2011 b.

Wakita, H., Geochemical challenge to earthquake prediction, Proc. Nat'1 Acad. Sci., USA, 93, 37813786, 1996.

Yang, S. S., Asano, T., and Hayakawa, M., Abnormal gravity wave activity in the stratosphere prior to the 2016 Kumamoto earthquakes, J. Geophys. Res., Space Phys., in press, 2019.

Yasuoka, Y., Nagahama, H., and Ishikawa, T., Anomalous Radon Concentration Prior to an Earthquake, LAP LAMBERT Acad. Publ., Beau Bassin, 138p, 2010. 